\title{
Modification of the Minimal Bergman Model of the "Insulin-Glucose" System and its Implementation in MatLab/Simulink
}

\author{
Belov Vladimir \\ Head of trhe Departament of Medical \\ Informatics and Cybernetics \\ Pskov State University \\ Pskov, Russian Federation \\ vsbb@yandex.ru
}

\author{
Alexander Samarkin \\ Associate Professor of the \\ Departament of Medical Informatics \\ and Cybernetics \\ Pskov State University \\ Pskov, Russian Federation \\ alexsamarkin@gmail.com
}

\author{
Mark Procofiev \\ Student of the Specialty Medical \\ Cybernetics \\ Pskov State University \\ Pskov, Russian Federation \\ mark04069@gmail.com
}

\author{
Tatyana Komandresova \\ Associate Professor of the Department \\ of Fundamental Medicine and \\ Biochemistry \\ Pskov State University \\ Pskov, Russian Federation \\ tatmyh005@mail.ru
}

\begin{abstract}
The article discusses a modification of Bergman's minimal mathematical model of the "insulinglucose" system, which allows simulating controlled exogenous sources of glucose and insulin into the patient's blood on the model and investigating the dynamics of changes in their concentrations in normal conditions, in type I DM and type II DM. A modeling scheme is presented in graphic notations of the MatLab / Simulink computer mathematics system and a number of computational experiments on it are described to determine the type of glycemic profiles of glucose and insulin concentration in the patient's blood in the noted situations. The fundamental possibility of using model mappings in the MatLab/Simulink environment for the study and tuning of the loop for automatic regulation of the "insulin-glucose" balance in the patient's blood using a controlled insulin pump is demonstrated. It was also found that the modified minimal model can be customized for a specific patient with diabetes, which makes it possible to use it to solve the problems of individual prediction of the development of a diabetic disease in a specific patient. In addition, the described model makes it possible to recreate and virtually investigate various conditions and cases on it that affect the dynamics of insulin and glucose concentrations in the patient's blood, for example, when he performs physically stressed activities, in the presence of the effects of "aging" of insulin-producing cells in the pancreas. iron, etc.
\end{abstract}

Keywords - Diabetes mellitus, Insulin-glucose balance, Glycemic monitoring, Computer simulation, Model personalization, Computational experiment.

\section{INTRODUCTION}

According to the World Health Organization (WHO), diabetes mellitus (DM) is currently a global problem on a planetary scale. The number of patients with diabetes in the world over the past 10 years has more than doubled, and is currently approaching 500 million people [1]. It is known [2] that DM is dangerous, first of all, due to its complications, in particular, such as: blindness, renal failure, heart attack, stroke, amputation of the lower extremities, etc. are still practically inevitable even if all recommendations of the attending physicians are followed [3]. Until now, there is not a single effective method of treatment to get rid of DM. It is for these reasons that such tasks are important as: increasing the effectiveness of diabetes prevention, ensuring highquality current monitoring of the condition of a patient with diabetes, performing operational prediction and managing the concentration of glucose and insulin in his blood. In this case, the formation of insulin therapy regimens for patients with DM is based on the experience 
Belov Vladimir et al. Modification of the Minimal Bergman Model of the "Insulin-Glucose" System and its Implementation in MatLab/Simulink

and intuition of a doctor and is more an art [4], a medico-creative process, rather than the result of deductive inferences based on objective formalized diagnostic characteristics of a diabetic disease and information about the individual characteristics of its course in specific patients. Note that in the empirical selection of the parameters of insulin therapy for diabetes, the doctor, fearing the occurrence of a hypoglycemic state in the patient, is often limited only by the weakening of hyperglycemia. At the same time, the patient's condition remains insufficiently compensated, which condemns him to subsequent late vascular complications. Here, one of the most effective ways to increase the doctor's confidence in the safety of the process of normalizing glycemia in a patient with diabetes and, accordingly, to prevent or at least significantly reduce the likelihood of developing complications from diabetes is to use computational research sites at the stage of determining the modes and parameters of insulin therapy, where the objects of study are individualized (i.e. customized for specific patients with diabetes) mathematical models of the dynamics of glucose and insulin in their blood.

The aim of this work is to construct and analyze the options for using a patient-oriented simulation computerized model for regulating the concentration of glucose and insulin in the blood of a patient with diabetes with the possibility of studying different types of this disease on it.

Note that in relation to the subject of this article, models and methods of conducting computer experiments with them are of interest, which describe the processes of ensuring the balance of "insulin-glucose" in the blood of a patient with diabetes. The need for just such models and the results of their research is due to the fact that in the treatment of DM, the primary and main task is to normalize blood glucose levels and prevent hypo- and hyper-glycemic conditions in a patient. Naturally, the presence of a correct formal description of the "insulinglucose" balance in the blood, adapted and adjusted for a particular patient, makes it possible to solve a number of clinical problems aimed at improving the health of a patient with diabetes. This is, firstly, the organization of continuous monitoring of the glucose level before and after its correction (if necessary); secondly, the modeling of the development of controlled endocrine and physiological processes using various schemes for correcting the insulin-glucose balance. In this case, the correction of the glucose concentration in the patient's blood can be manual in nature (then the task of the model system is to predict the conditions of potentially unfavorable development of processes and to warn the patient about the need for correction), or it can be carried out semi- or completely automatically (by using devices for minimally invasive exogenous insulin delivery with a guided insulin pump). In addition, the process of external insulin delivery (bolus, basal, or combined) and the patient's body response to such an effect should also be investigated under model conditions, at least for the correct calculation of the parameters of external insulin exposure.

\section{LITERATURE REVIEW}

From a mathematical point of view, biological functional systems, which include systems for regulating blood glucose concentration, are stochastic nonlinear systems with multi-compartment interactions [5]. Such systems include many interacting components at the organ, tissue, cellular and subcellular levels, reflecting the dynamic processes of controlling changes in the concentration of glucose and insulin in the blood plasma. simplifiedly called the "insulin-glucose" system.

When creating mathematical model constructions and schemes that allow performing computational experiments in order to predict the dynamics of changes in glucose and insulin concentrations in the blood of a patient with diabetes and to solve problems of their control, the following approaches are used [6, 7]:

1). Empirical or "black box" models that use real physiological and other data about the patient's condition that can, to varying degrees, affect the controlled levels of glucose and insulin in the blood of a patient with diabetes - these are the previous values of glucose and insulin concentrations, physiological parameters organism (weight, temperature, blood pressure, cardiac parameters, blood composition, etc.), properties of exogenous sources of glucose and insulin intake, etc. As basic mathematical representations, analytical schemes based on linear autoregressive mappings are used [8, $9,10]$ and neural network mathematical constructions $[11,12]$.

2). Theoretical or "complete" models, based primarily on the laws of physiology, built without involving additional empirical assumptions, - they describe physiological and pathophysiological processes occurring at the organ, tissue, cellular and subcellular levels, including the distribution and dynamic changes in glucose concentration and insulin in various organs and tissues of the body, the processes of absorption of exogenous glucose, its utilization and elimination, natural (in the pancreas) and artificial (from exogenous sources) mechanisms of insulin production, etc. Formal descriptions of complete models are based on compartment mappings and the mathematical apparatus of ordinary differential equations (ODE) [13, 14, 15], including ODE with delay $[16,17]$, as well as the apparatus of integrodifferential formal notations [18] and graph theory [19].

3). Mixed or semi-empirical models using somewhat simplified mathematical constructions of theoretical models of key physiological and pathophysiological processes occurring at the organ and tissue levels and determining the conditions and mechanisms of regulation of carbohydrate metabolism in the patient's body, supplemented by empirical formed parameters, 
characteristics and functions. The empirical components introduced into the formal model schemes make it possible to reproduce in simulation conditions the functions of exogenous intake of glucose and insulin into the patient's body [20, 21], including variants with the function of controlling the concentration of glucose in the blood [22, 23], the mechanisms of the relationship between blood glucose and subcutaneous adipose tissue [24], characteristics reflecting changes in glucose concentration in the interstitial space [25], factors that take into account the effect of physical activity on the functioning of the insulin-glucose system [26], etc.

Analyzing the mentioned model concepts, it should be noted that not all the noted formal structures are applicable for individual adaptation for specific patients, for organizing guided insulin therapy, and also for setting model notations for patients with different types of diabetes.

Thus, empirical models based on autoregressive structures make it possible to effectively solve the problem of predicting changes in the level of glucose in a patient's blood, since according to the analysis of time series of real measurements of its concentration, it is possible to generate a forecast of their future values based on known data from the past. It should be noted here that such models make it possible to predict with a sufficiently high reliability the concentration of glucose in the blood only for the near future - from 30-45 minutes [8.9] to 4 hours [10]. These models have proven themselves well when customizing them for specific patients with type 1 diabetes and without diabetes, but they are not very effective in recreating type 2 diabetes. Also, autoregressive formal schemes are not sufficiently adapted to the reproduction of exogenous sources of glucose and insulin intake, to the implementation of blood glucose control regimes, to taking into account other physiological factors in the regulation of carbohydrate metabolism in the blood.

Neural network empirical models are also generally quite efficiently tuned to specific patients and make it possible to predict future glucose levels $[11,12]$ in his blood based on several previous values and / or some currently existing factors regulating carbohydrate metabolism. At the same time, it should be borne in mind that neural network models, before using them, require a training procedure, i.e. in fact, individualized adaptationadjustment of the model for a specific patient, and this procedure is rather complicated and time-consuming, which significantly hinders its widespread use both in solving research problems and in the medical practice of an endocrinologist. Also, neural network model structures are rather difficult to adapt to the tasks of automating insulin therapy in patients with diabetes.

Theoretical model representations use equations characterizing physiological and pathophysiological processes associated with the insulin-glucose system, written, if possible, on the basis of "first principles" ("First principles" are natural laws of nature that allow to substantiate any phenomenon without additional empirical assumptions or special models). This approach leads to the need to use in formal schemes high-order systems of ordinary (often nonlinear) differential, integro-differential, differential-graph equations. As examples of such models, let us mention J.T. Sorensen [27] (edited by researchers Parker R.S., Doyle F.J. et al. [28,29]), C. Cobelli et al. [30,31], Archimedes [32,33]. These models contain from 7 to 30 differential equations describing the mechanisms of glucose production and absorption, insulin release and utilization, and other physiological and pathophysiological processes that affect the "insulin-glucose" balance in the patient's blood. All noted models allow their customization for specific patients with diabetes, provide the opportunity to conduct model studies of all known types of diabetes (primarily types 1 and 2), make it possible to effectively predict the dynamics of changes in the concentration of glucose in the blood of a patient with diabetes in various life situations, and also on these models it is possible to recreate exogenous sources of glucose and insulin, incl. working in an automatic mode of insulin therapy for a patient with diabetes. However, the high and often excessive complexity of the development of such models, the significant difficulties in collecting and determining the values of biological variables that must be taken into account in their formal notations, prevent the widespread introduction of these model concepts into the clinical practice of endocrinologists.

In this regard, semiempirical models of the insulinglucose system are the most convenient for implementation in medical practice for treating patients with diabetes. As a rule, mixed models are a system of 23 , less often 4-6 nonlinear differential and / or integrodifferential equations of the first order, on the right side of which there are a number of components reflecting the mechanisms of production, exogenous intake, utilization, elimination of glucose and insulin, as well as, if necessary, recreating the influence of other physiological parameters of the body associated with carbohydrate metabolism. Among the semiempirical models of the insulin-glucose system, we note the following:

1) The minimal model (Bergman R.N. [14], which has three ODEs (equations for the dynamics of glucose, insulin and an auxiliary variable describing the dependence of the concentration of insulin in the blood on the amount of absorbed glucose).

2) Model Sturis J. [13], which includes six TACs and is based on two negative feedbacks describing the absorption of glucose by insulin and the release of insulin by the pancreas depending on the amount of glucose supplied.

3) Three-piece model Nikita K.S. [34], in the composition of three TACs - two TACs for the dynamics of insulin absorption (ultrashort and shortacting) and one TAC of changes in the concentration of glucose in the patient's blood caused by the last meal. 
4) Bennett D.L. and Gourley S.A. [13,16], in which systems of two ODEs with a lagging argument are used to describe the dynamics of glucose and insulin. These models subsequently underwent many modifications and refinements, which made them more universal and flexible to the real conditions of insulin therapy for patients with diabetes. These are the already mentioned models with exogenous sources of glucose and insulin [20,21], with components of semi-automatic control of patient's insulin therapy [22,23], which made it possible to significantly increase the level of adequacy of empirical models (especially Bergman R.N. medtli) under conditions of type 1 diabetes ... These are models that take into account the mechanisms of the relationship between the blood glucose content and subcutaneous adipose tissue [24], reflecting factors influencing the change in the glucose concentration in the interstitial space [25], etc.

Analysis of the mathematical structures of the mentioned mixed models showed that almost all of them can be used for the tasks of predicting changes in the concentration of glucose in the blood at different stages of the breakdown of diabetes disease in a patient, as well as with exogenous regular or irregular intake of glucose into the patient intravenous intake of insulin into the body of a patient with diabetes, incl. when using an insulin pump controlled by feedback from blood glucose sensors. However, among the semiempirical model representations of the carbohydrate balance system in the patient's blood, the Bergman R.N. [14] and its modifications are, perhaps, the most convenient for reconstruction by means of computer mathematics due to the moderate complexity of the mathematical representation of this model, the high flexibility of its analytical design, and the good adaptability of the formal scheme of the model to the implementation of control actions.

\section{MATERIALS AND METHODS}

As software tools necessary for the development and construction of a model range for carrying out computational experiments with models of the insulinglucose system, computer mathematics software systems, such as MapleModeller or SciLabXCos, can be used. However, the Matlab software system of computer mathematics (Matrix Laboratory) [35] with the Simulink extension possesses the best tools for simulation computational modeling. This environment is equipped with a good high-level language and an interactive environment for graphical visual programming, numerical calculations and visualization of the results of computational experiments with multi-domain dynamic systems and processes.

It is known [36] that an increased blood glucose level can be initiated by the following reasons:

a) insufficient production of insulin by $\beta$-cells of the pancreas; b) the molecular structure of insulin is changed ("defective" insulin);

c) insulin is not recognized by cell receptors.

In case of insufficient insulin production (case (a)) diabetes mellitus is called insulin-dependent, or type I diabetes, in cases (b) and (c), when enough insulin is produced, but it is not recognized or is "defective", diabetes is called non-insulin dependent, or type II diabetes.

Let us introduce a number of starting points:

1) There is a certain patient-specific "nominal (normal, acceptable) level" of glucose concentration in the blood $G_{0}$.

2) If the current glucose concentration exceeds the value $G_{0}$, i.e. when $G(t)>G_{0}$, insulin is produced in proportion to the difference $\left(G(t)-G_{0}\right)$ (often with some time delay).

3) Insulin is a conductor of glucose through the cell membrane, thus mutual utilization (elimination) of insulin and glucose occurs in proportion to the product of their concentrations in the blood.

4) When the level of glucose concentration in the blood falls below the level $G_{0}$, i.e. when $G(t)<G_{0}$, there is a release of glucose from the liver in proportion to the difference $\left(G_{0}-G(t)\right)$.

5) After exceeding the current concentration of glucose in the blood of a certain upper value $G_{c r}$, i.e. when $G(t)>G_{c r}$, glucose is excreted from the body through the kidneys.

6) Insulin circulating in the blood can be in two forms in a free state and associated with proteins. Hereinafter, insulin will always be understood as free insulin.

Based on the above and modernized equations of the Bergman minimal model. system "glucose-insulin", given in [21, 37], we will form the following system of nonlinear differential equations characterizing the dynamics of changes in glucose and insulin in the blood of a patient with diabetes:

$$
\left\{\begin{array}{l}
\frac{d I(t)}{d t}=\alpha\left(G_{0}^{-}\right) \theta\left(G_{0}^{-}\right)-\eta G(t) I(t)+L(t) \\
\frac{d G(t)}{d t}=\gamma\left(G_{0}^{+}\right) \theta\left(G_{0}^{+}\right)-v G(t) I(t)-\mu\left(G_{c r}^{-}\right) \theta\left(G_{c r}^{-}\right)+S(t)
\end{array},\right.
$$

where $G(t), G_{0}, G_{c r}$ - the previously mentioned designations; $G_{0}^{-}=G(t)-G_{0}, G_{0}^{+}=G_{0}-G(t), \quad G_{c r}^{-}=G(t)-G_{c r}$; $I(t)$ - the concentration of insulin in the blood at a point in time $t ; \alpha$-coefficient of insulin production by $\beta$-cells of the pancreas at $G(t)>G_{0} ; \eta$-coefficient of utilization of insulin by glucose; $\gamma$ - coefficient of glucose withdrawal from the liver to maintain its normal level in the blood; $v$ - coefficient of glucose utilization by insulin; $\mu$ - parameter of elimination of glucose through 
the kidneys at $G(t)>G_{c r}$; (note that the values $\alpha, \eta, \lambda, v, \mu$ are personalized values); $L(t)$ - a function that takes into account the external supply of insulin; $S(t)$ - a function that takes into account the flow of glucose into the patient's blood (for example, as a result of his nutrition); $\theta(x)$ - Heaviside function of the form:

$$
\theta(x)=\left\{\begin{array}{l}
0, \text { if } x \leq 0 \\
1, \text { if } x>0
\end{array},\right.
$$

where one of the following values is used as an argument: $x \in\left[G_{0}^{-}, G_{0}^{+}, G_{c r}^{-}\right]$.

Using the graphic notations of the MatLab / Simulink environment, we will form the following model constructions of the equations of system (1) - a model of the dynamics of insulin changes (Fig. 1) and a model of the dynamics of glucose changes (Fig. 2).

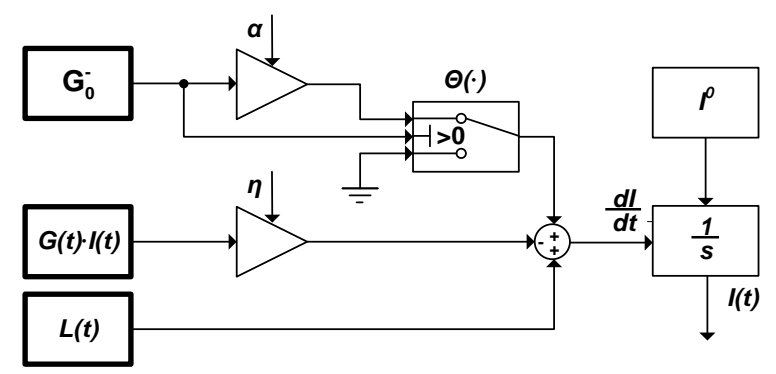

Fig. 1. Model of the dynamics of changes in insulin.

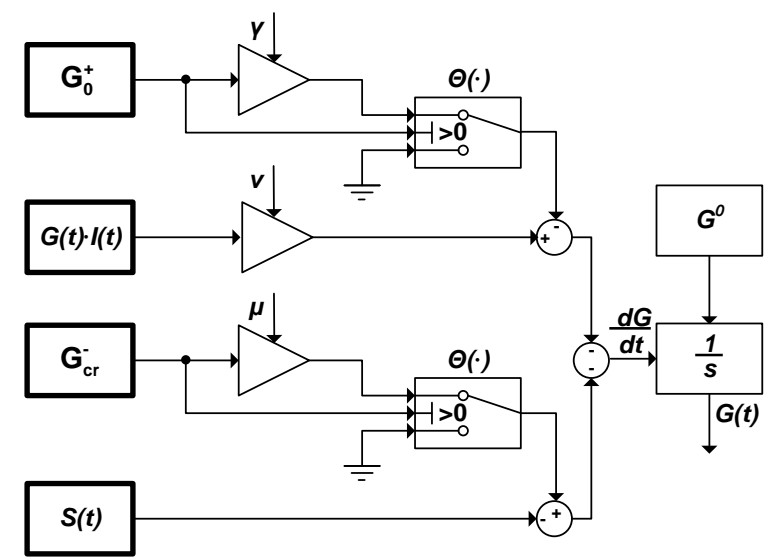

Fig. 2. Model of the dynamics of changes in glucose.

In these structures: block « $\frac{1}{\mathrm{~s}} »-$ integrator; blocks «I $\mathbf{I}^{0} »$ and $\left\langle\mathbf{G}^{0} »-\right.$ setters of the initial conditions of the integrator; block " $D$ » - multiplier by the corresponding coefficient $\alpha, \eta, \gamma, v$ or $\mu$; subblocks « $\mathbf{G}_{0}^{-}$", «L(t)», «G(t) $\cdot \mathbf{l}(\mathbf{t}) »,\left\langle\mathbf{G}_{0}^{+} »,\left\langle\mathbf{G}_{\mathrm{cr}}^{-} »,\langle\mathbf{S}(\mathbf{t}) »\right.\right.$ are intended to form the corresponding elements of the first equation system (1).

To determine the regularities recreated in the « $\mathbf{S}(\mathbf{t})$ » and «L(t)» subunits, simulating external sources of glucose and insulin intake, let us set out mathematical descriptions of the processes of glucose and insulin intake.
Thus, a single act of glucose intake into the patient's body from an exogenous source is modeled by a nonnegative and one-extreme Gaussian function [37]:

$$
S^{(\mathbf{0})}(t)=A_{s}^{(0)} \exp \left(-\frac{\left(t-T^{(0)}\right)^{2}}{2 \sigma^{2}}\right),
$$

where $A_{s}^{(0)}$ - the amplitude, which determines the maximum value of the peak of the injection of glucose into the blood after a meal; $T^{(\mathbf{0})}$ - the interval defining the moment of the beginning of feeding (the beginning of exogenous glucose intake) relative to the beginning of the model experiment (this moment corresponds to $t=0$ ); $\sigma$ - variance, which controls the width of the peak, i.e. in fact, the rate of assimilation of food by the patient, the full dose of which is $A_{s}^{(0)} \sigma^{2}$ [21], while it is obvious that $95 \%$ of the food (respectively, $95 \%$ of exogenous glucose) will be assimilated by the body in an interval equal to $2 \sigma$. The value of the variable corresponding to the maximum of the function $S^{(0)}(t)$ is determined by the formula:

$$
t_{\max }^{(0)}=\left.t\right|_{s^{(0)}(t)=s_{\max }^{(0)}}=\left(T^{(0)}+\sqrt{\left(T^{(0)}\right)^{2}+4 \sigma^{2}}\right) / 2 .
$$

It is believed [21] that the food taken by the patient is completely converted into glucose on average within two hours after the beginning of the feeding process, i.e. the moment $t_{\max }^{(\mathbf{0})}$ is 1 hour from the start of the meal $\left(t-T^{(\mathbf{0})}\right)$.

Three meals a day (three daily exogenous intake of glucose into the patient's body), provided that the daily meal is equivalent to two morning or evening meals, can be described as follows [37]:

$$
S^{c}(t)=A_{S}^{(0)}\left(\psi\left(T^{(\mathbf{1})}\right)+2 \psi\left(T^{(2)}\right)+\psi\left(T^{(3)}\right)\right),
$$

where $\psi\left(T^{(\mathbf{i})}\right)=\exp \left(-\frac{\left(t-T^{(\mathbf{i})}\right)^{2}}{2 \sigma^{2}}\right), i=1,2,3$; at the same time, as a first approximation, we can assume that the rate of assimilation of food by the patient is the same in all periods of food intake.

To set a formal description of the subblock «L(t)», which simulates an exogenous source of insulin intake, let us take into account that the need to forcibly inject insulin into the patient's blood arises when the patient has diabetes mellitus and has certain difficulties with blood. There are two ways of getting insulin into a patient's blood from an external source.

The first method involves the introduction of insulin into the blood in a volume proportional to the value of the difference $\left(G(t)-G_{0}\right)$, moreover, only after revealing the fact of such an excess. Mathematically, this method is described by the following relationship: 
Belov Vladimir et al. Modification of the Minimal Bergman Model of the "Insulin-Glucose" System and its Implementation in MatLab/Simulink

$$
L_{1}(t)=\left\{\begin{array}{l}
k_{I}\left(G(t)-G_{0}\right), \text { if }\left(G(t)-G_{0}\right)>0 \\
0, \text { if }\left(G(t)-G_{0}\right) \leq 0
\end{array},\right.
$$

where $k_{\mathrm{I}}$ - is the coefficient of the relationship between glucose and insulin concentrations.

The second method is based on a relay mechanism for controlling the process of insulin intake into the patient's body, which is triggered when the moment the condition is met $G(t)-G_{0}>G^{*}$, where $G^{*}$ - is some permissible excess of the minimum glucose concentration $G_{0}$. From this moment, insulin is supplied into the patient's blood at a constant concentration $G_{0}$. Mathematically, this method of injecting insulin is described by the expression:

$$
L_{2}(t)=\left\{\begin{array}{l}
L^{*}, \text { if }\left(G(t)-G_{0}\right)>G^{*} \\
0, \text { if }\left(G(t)-G_{0}\right) \leq G^{*}
\end{array} .\right.
$$

In both schemes of organizing the supply of exogenous insulin into the blood of a patient with diabetes, the supply is stopped as soon as the condition for the start of its administration is stopped.

\section{RESULTS}

Here are some of the results of computational experiments on the simulation test site of the "insulinglucose" system, assembled on the basis of the structural structures shown in Fig. 1 and Fig. 2. The purpose of the experiments is to determine the establishment of daily glycemic profiles of glucose and insulin in the patient's blood in the situations:

Situation 1. The patient is healthy. The function of insulin production by the $\beta$-cells of the pancreas is normal. Excess blood glucose is promptly neutralized by insulin produced by the body in the right amount.

Situation 2. The patient has type I diabetes mellitus. There is an insufficient level of insulin production by $\beta$ cells of the pancreas. Correction of the glucose-insulin balance in the patient's blood is not performed.

Situation 3. The patient has type II diabetes mellitus. The pancreas makes the required amount of insulin, but this insulin is "defective". Correction of the "glucose-insulin" balance in the patient's blood is not fully carried out.

Situation 4. The patient has type I diabetes mellitus and there is a controlled system for introducing exogenous insulin into his blood. There is an insufficient level of insulin production by the pancreas or insulin is not produced in it at all. There are means for continuous monitoring of blood glucose concentration and a system for automatic correction of it by forcibly injecting insulin into the blood from an exogenous source.
Now let us set the initial parameters necessary for organizing correct modeling (partially taking into account the data given in [21, 37]):

1) Periods of simulation: 1 day (24 hours).

2) The number of patient meals (glucose intake from an exogenous source) per day is 3 with the following time stamps: 08-00, 14-30, 19-30 hours.

3) Daily markers of the patient's blood glucose maxima: 09-00, 15-30, 20-30 hours.

4) Concentrations of glucose in the patient's blood:

- before meals (on an empty stomach): 3,3 ... 6,1 $\mathrm{mMol} / \mathrm{l}$;

- after each meal (after 1 hour): 8,9 mMol/l;

- minimum allowable: 3,3 mMol/l;

- nominal (normal): $5 \mathrm{mMol} / \mathrm{l}$;

- critical: $10 \mathrm{mMol} / \mathrm{l}$.

5) The insulin concentration in the patient's blood varies from 0 to $170 \mathrm{mU} / \mathrm{ml}$ ( $\mathrm{mU}$ - milli units of insulin).

6) Typical values of the coefficients in the equations of system (1), independent of the situations under consideration:

- coefficient of utilization of insulin by glucose;

- coefficient of glucose output from the liver;

- coefficient of glucose excretion through the kidneys.

7) Coefficient of insulin production by $\beta$-cells of the pancreas: situations 1 and $3-\alpha=15$; situations 2 and $4-\alpha=3$.

8) Coefficient of glucose utilization by insulin: situations 1,2 and $4-v=6$; situation $3-v=1$.

Here are some results from computational experiments.

Situation 1. The patient is healthy. The blood glucoseinsulin balance is normal. The glycemic profiles of glucose and insulin concentrations have the form shown in Fig. 3, and their daily dynamics corresponds to the clinical data of a healthy organism.

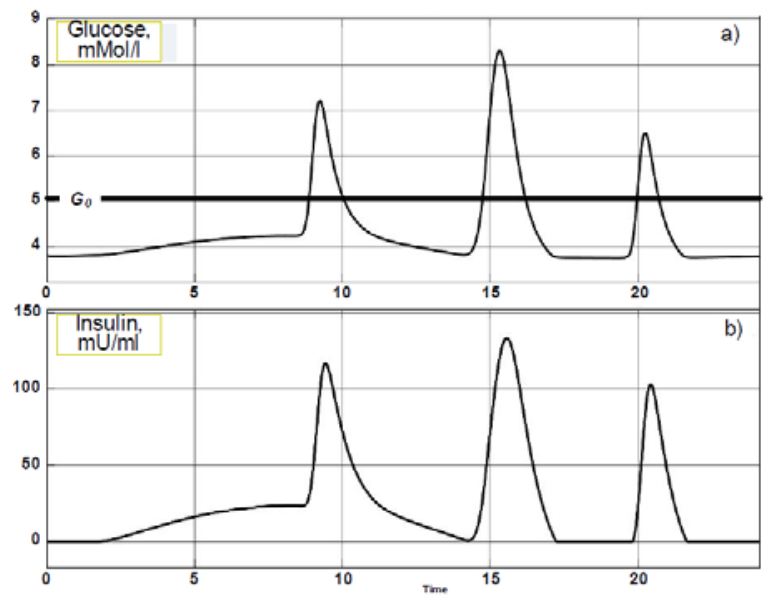

Fig. 3. Daily glycemic profiles of glucose (a) and insulin (b) concentrations in the blood of a healthy patient. 
The blood glucose level does not exceed the critical value of $10 \mathrm{mMol} / \mathrm{l}$. In the first approximation, the selected values of the coefficients in model (1) can be considered correct. Then, by manipulating the values of these coefficients, one can expect physiologically correct results from the model for certain disorders of carbohydrate metabolism. However, this does not replace the mandatory identification and adjustment procedures for the model for specific patients when using it in clinical practice.

Situation 2. A patient has type I diabetes mellitus. The insulin produced by the patient's body is not sufficient to eliminate glucose. In this case, the glycemic profiles of glucose and insulin concentrations in the blood take the following form - see Fig. 4. Insufficient insulin level leads to the fact

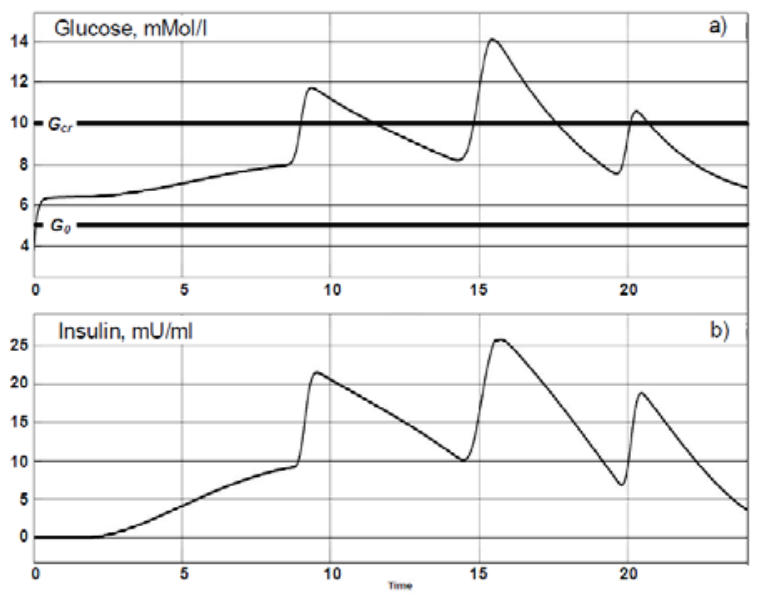

Fig. 4. Daily glycemic profiles of glucose (a) and insulin (b) concentrations in the blood of a patient with type I diabetes.

that after each meal there is a rapid increase in the concentration of glucose in the patient's blood, exceeding the critical level of $10 \mathrm{mMol} / \mathrm{l}$. Then the glucose concentration slowly decreases, but does not reach the normal value $5 \mathrm{mMol} / \mathrm{l}$ and remains at a sufficiently high level. This effect naturally leads to the fact that excess glucose from the body's blood is excreted through the kidneys. This situation is characterized by blood effects typical of type I diabetes.

Situation 3. The patient has type II diabetes mellitus. The pancreas of the patient's body produces insulin in sufficient quantities, but it does not have the necessary properties for efficient utilization of glucose, i.e. is "defective". The glycemic profiles of glucose levels and moderately "defective" insulin in the blood are as follows - see fig. 5 . It can be seen that, as in the case of diabetes mellitus I, after each meal there is a rapid increase in the concentration of glucose in the blood of a patient with diabetes mellitus II; however, insulin utilization requires $20-30 \%$ more insulin than in a healthy person. organism (Fig. 3). In addition, there is a slowed down, compared with the norm, process of lowering the blood glucose level and reaching its normal value only by the end of the patient's daily activity period (by 00 hours).

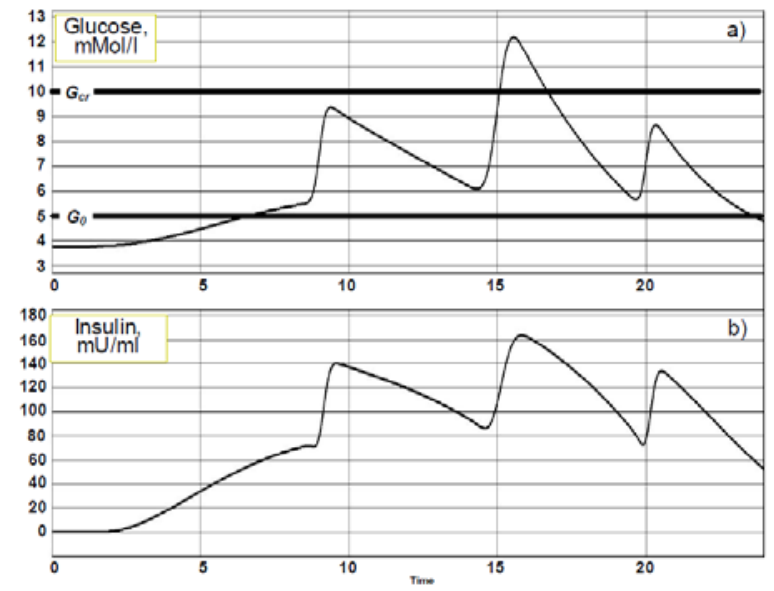

Fig. 5. Daily glycemic profiles of glucose (a) and insulin (b) concentrations in the blood of a patient with type II diabetes (a case with moderately "defective" insulin).

Situation 4. A patient has type I diabetes mellitus and there is a controlled system for introducing exogenous insulin into his blood. There are means of continuous monitoring of the patient's blood glucose level and a controlled system for continuous delivery of insulin into the blood from an exogenous source, which is equipped with an insulin pump [38]. Let the proportional pump control mode be selected in accordance with expression (6). The coefficient of the relationship between the concentrations of glucose and insulin is assumed to be equal $k_{\mathrm{I}}=22$, since it is generally accepted [21] that 1 Unit of insulin capable of utilizing $22 \mathrm{mMol}$ glucose. Then the glycemic profiles of glucose and insulin concentration in the patient's blood take the form shown in Fig. 6. Here it is necessary to pay attention to the fact that in a patient with type I diabetes mellitus, in the absence of an automatic system for correcting the glucose-insulin balance in the blood by exogenous insulin, the maximum permissible glucose concentration level of $10 \mathrm{mMol} / \mathrm{l}$ is exceeded after each meal by the patient (see Fig. 4.a).

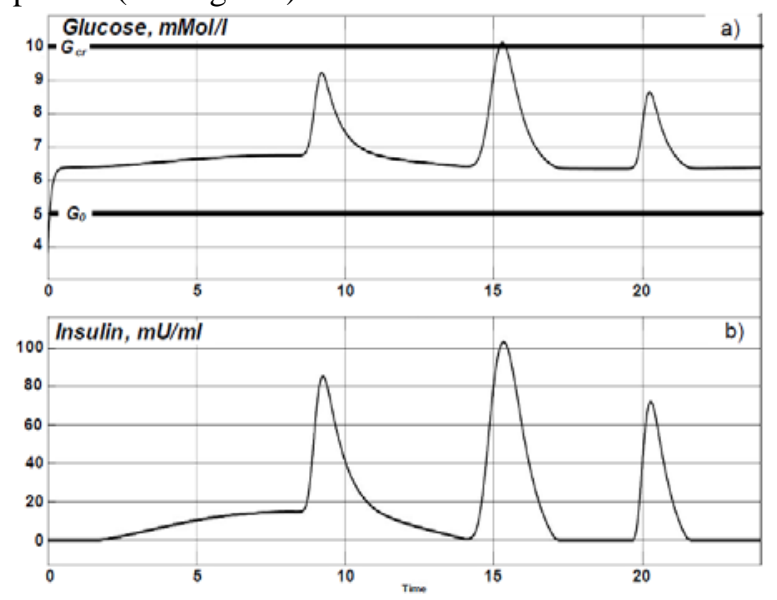

Fig. 6. Daily glycemic profiles of the concentration of glucose (a) and insulin (b) in the blood of a patient with type I diabetes mellitus, provided with a continuous supply of exogenous insulin with a proportional regulator of its consumption. 
When such a patient enters the bloodstream of insulin from a controlled external source, the concentration of glucose in his blood exceeds the maximum allowable value of $10 \mathrm{mMol} / \mathrm{l}$ only once - about $15-30$ in the afternoon for a relatively short time after the day (maximum) food intake by the patient, which gives the maximum injection exogenous glucose into the body (Fig. 6.a).

\section{DISCUSSION}

So, the considered mathematical model of the "insulin-glucose" balance (system of equations (1)), despite the undefined "roughness" of the formal description and its model representations using MatLab / Simulink, as well as the results of computational experiments carried out on this model, demonstrate the following dynamics changes in the model concentrations of glucose and insulin in the patient's blood, which fully corresponds to the real glycemic profiles of the concentrations of glucose and insulin in the blood of the human body, both for its healthy state and in the presence of type I and II diabetes mellitus. Thus, the model dynamic sections of the diurnal processes of changes in the level of glucose and insulin in the blood of a healthy patient presented in Fig. 3 adequately converge with the dynamical processes of the same name obtained in real examinations of practically healthy people (see, for example, [39]). Model glycemic profiles of glucose and insulin concentration in the blood of a patient with type I diabetes mellitus (Figs. 4 and 6) also identically reflect the dynamics of real analogous profiles obtained during glycemic control of patients with type I diabetes (see data in $[39,40])$. The calculated profiles of changes in glucose and insulin levels obtained for a patient with type II diabetes (Fig. 5) also provide good agreement with the real results of daily glycemic monitoring in patients with type II diabetes (see, for example, [41]).

It should be noted that the described model of the "glucose-insulin" balance in the patient's blood has good potential for its further improvement.

So, it is of interest to model such behavior of the main organ-generator of insulin (pancreas), which reflects its dynamic "fatigue", ie. allows to recreate in model conditions a variable rate of insulin production with limitation of the upper limit of such production. To simulate this situation, it is sufficient in the first equation of system (1) to make the constant coefficient of insulin production variable, depending on the temporal characteristic or other changing factors.

Replacing the coefficients and, which determine the processes of mutual utilization of insulin and glucose in the corresponding equations of system (1) by functional operators that take into account the rate of glucose consumption under the influence of physical exertion on the patient's body, it is possible to reproduce on the considered model of carbohydrate metabolism various "insulin-glucose" consequences in the patient's blood from his hard work, physical exercise, sports and other similar physically stressful activities.
The model of insulin-glucose balance in the patient's blood described in this article can be generally recommended for implementation in clinical practice to improve the quality of insulin therapy in patients with diabetes mellitus, provided that the issues of individual identification and adjustment of the mathematical description of carbohydrate metabolism for specific patients with diabetes mellitus are resolved. The formal construction of the model description of the insulinglucose system in the patient's blood (equations of system (1)) allows it to be adjusted for a specific patient by adjusting the values of the coefficients of insulin production by the pancreas $(\alpha)$, mutual utilization of insulin and glucose $(\eta, v)$, and glucose output from the liver $(\gamma)$, elimination of glucose through the kidneys $(\mu)$, as well as adjusting the values of the nominal $G_{0}$ and critical $G_{c r}$ levels of glucose concentration. This significantly increases the flexibility and scope of the considered model, makes it "patient-oriented", which is especially important in the case of its introduction into the clinical practice of an endocrinologist in order to study and predict the development of a diabetic disease in a particular patient.

Also, the methods and mechanisms of organizing glycemic monitoring of blood glucose levels with the automation of the process of exogenous insulin entering it using a controlled insulin pump also require some improvement. Here it is possible to propose a wider use of the existing developments in this area - see, for example, [38,42], which describes systemic approaches to the organization of glycemic control of the blood state of a patient with type I diabetes mellitus and systemic issues of building an insulin pump with its control circuit.

Obviously, the modified Bergman minimal model of the "insulin-glucose" system in the patient's blood considered in this article makes it possible to recreate and study under model conditions various clinical aspects of carbohydrate metabolism both for a healthy patient's body and in the presence of pathologies, including diabetes mellitus I and II types, changes in the balance of "insulin-glucose" during physical exertion, the effects of "aging" of the insulin-production capacity of $\beta$-cells of the pancreas.

\section{CONCLUSION}

The authors have shown the possibility of using modern software tools - the MatLab/Simulink computer mathematics system, initially focused on solving various dynamic problems of scientific, technical, statistical, optimization focus using the mechanisms of simulation modeling and the theory of experiments, to build dynamic simulation models of carbohydrate metabolism processes in the blood a patient suffering from diabetes mellitus and conducting research on this model. The fundamental possibility of using the MatLab/Simulink software system for organizing controlled computational experiments on a model range of the insulin-glucose system, as well as for high-quality visualization of the key dynamic characteristics of the studied processes of 
carbohydrate metabolism in the blood of a patient with diabetes mellitus, has been demonstrated. The obtained model results showed good agreement with real glycemic profiles of insulin and glucose concentrations in the blood of a healthy patient and in the presence of diabetes mellitus.

Thus, this article shows how an endocrinologist can effectively use the MatLab/Simulink software environment as a solid modern clinical research tool aimed at studying the dynamics of the development of signs of pathological processes associated with carbohydrate metabolism in order to ultimately improve the quality and efficiency diabetes mellitus treatment.

\section{VII.REFERENCES}

[1] Standards of specialized diabetes care. Edited by Dedov I.I., Shestakova M.V. and Mayorov A.Yu. 9th Edition. Moscow: 2019,144 p. https://doi.org/10.14341/DM221S1. (in Russian)

[2] S.N. Okulova, Review of mathematical models of the dynamics of insulin and glucose. Materials of the XI International Student Scientific Conference "Student Scientific Forum-2019". 2019. [Online].

Available: https://scienceforum.ru/2019/article/2018012293 [Accessed Mart, 14, 2021]. (in Russian)

[3] F., Cosentino, P.J. Grant, V. Aboyans and etc., ESC/EASD Guidelines for Diabetes Mellitus, Prediabetes and Cardiovascular Disease, Russian journal of cardiology, 2020, vol. 25, no4, p. 3839. ttps://doi.org/10.15829/1560-4071-2020-3839 (in Russian)

[4] Definition, Diagnosis and Classification of Diabetes Mellitus and its Complications: Report of a WHO Consultation. Part 1: Diagnosis and Classification of Diabetes Mellitus. Geneva: WHO. Department of Noncommunicable Disease Surveillance, 1999, $59 \mathrm{p}$.

[5] F.J. Doyle, L. Jovanovic, D.E. Seborg, R.S. Parker and B.W. Bequette, A tutorial on biomedical process control, J. Process Control., 2007, vol. 17, pp. 571-572. https://doi.org/10.1016/j.jprocont.2007.01.012.

[6] N.P. Balakrishnan, G.P. Rangaiah and L. Samavedham, Review and analysis of blood glucose (BG) models for type 1 diabetic patients,. Ind. Eng. Chem. Res., 2011. vol. 50, no. 21, pp. 1204112066. https://doi.org/10.1021/ie2004779.

[7] V.A. Karpel'ev, Yu.I. Filippov, Yu.V. Tarasov, M.D. Boyarsky, A.Yu. Mayorov, M.V. Shestakova and I.I. Dedov, Mathematical Modeling of the Blood Glucose Regulation System in Diabetes Mellitus Patients, Vestnik RAMN, 2015, vol. 5, pp. 549-60. https://doi.org/10.15690/vramn.v70.i5.1441. (in Russian)

[8] T. Bremer and D.A. Gough, Is blood glucose predictable from previous values? A solicitation for data, Diabetes, 1999, vol. 48, pp. 445-451. https://doi.org/10.2337/diabetes.48.3.445.

[9] M. Eren-Oruklu, A. Cinar, L. Quinn and D. Smith, Adaptive control strategy for regulation of blood glucose levels in patients with type 1 diabetes, J. Process. Control., 2009; 19: 1333-1346. https://doi.org/10.1016/j.jprocont.2009.04.004.

[10] T. Van Herpe, M. Espinoza, B. Pluymers, P. Wouters, F. De Smet, G. Van Berghe and B. De Moor, Development of a critically ill patient input output model. Proceedings 14th IFAC Symposium on System Indentification (SYSID 2006), Newcastle, Australia, 2006, pp. 482-486. https://doi.org/10.3182/20060329-3-AU2901.00073.

[11] S.G. Mougiakakou, A. Prountzou, D. Iliopoulou, K.S. Nikita and W. Vazeou, Neural network based glucose - insulin metabolism models for children with Type 1 diabetes, IEEE Eng. Med. Biol. Soc., $\quad 2006 ; \quad 1: \quad 3545 \quad-3548$. https://doi.org/10.1109/IEMBS.2006.260640.

[12] N. Auwal, D.M. Hamman, G. Ibrahim and M.J. Abdullahi, Adaptive Neuro-Fuzzy System to Determine the Blood Glucose Level of Diabetic,. Mathematics and Computer Science, 2019, vol. 4, no. 3, p. 63-67 https://doi.org/10.11648/j.mcs.20190403.11.
[13] A. Makroglou, J. Li and Y. Kuang, Mathematical models and software tools for the glucoseinsulin regulatory system and diabetes: an overview, Proceedings of the 2005 IMACS. Applied Numerical Mathematics, 2006, vol. 56, no. 3-4, pp. 559-573. https://doi.org/10.1016/j.apnum.2005.04.023.

[14] R.N. Bergman, Pathogenesis and prediction of diabetes mellitus: Lessons from integrative physiology, in: Irving L. Schwartz Lecture, Mount Sinai J. Medicine., 2002, vjol. 60, pp. 280-290.

[15] I.M. Tolich, E. Mosekilde and J. Sturis, Modeling the insulinglucose feedback system: The significance of pulsatile insulin secretion, J. Theor. Biol., 2000, vol. 207, pp. 361-375. https://doi.org/10.1006/jtbi.2000.2180.

[16] D.L. Bennett and S.A. Gourley, Asymptotic properties of a delay differential equation model for the interaction of glucose with plasma and interstitial insulin, Appl. Math. Comput., 2004, vol. 151, pp. 189-207. https://doi.org/10.1016/S0096-3003(03)003321.

[17] K. Engelborghs, V. Lemaire, J.Bélair and D. Roose, Numerical bifurcation analysis of delay differential equations arising from physiological modeling, J. Math. Biol., 2001, vol. 42, pp. 361385. https://doi.org/10.1007/s002850000072.

[18] A. De Gaetano and O. Arino, Mathematical modelling of the intravenous glucose tolerance test, J. Math. Biol., 2000, vol. 40, pp. 136-168.

[19] A.G. Borzov A.V., Dreval and S.I. Mukhin, Modeling of blood glucose dynamics with account of systemic loop topology, Mathematical modeling, 2015, vol. 27, no. 2, pp. 3-24. (in Russian)

[20] M.E. Fisher, A semiclosed loop algorithm for the control of blood glucose levels in diabetics. IEEE Transact. Biomed. Engineering, 1991, vol. 38, no. 1, pp. 57-61. https://doi.org/10.1109/10.68209.

[21] N.A. Shirokova, Mathematical modeling of glucose and insulin sources in the insulin-glucose balance model, Mathematical structures and modeling, 2004, vol. 14, pp. 47-52. (in Russian)

[22] T. Van Herpe, B. Pluymers, M. Espinoza, G. Van den Berghe and B. De Moor, A minimal model for glycemia control in critically ill patients, IEEE Eng. Med. Biol. Soc., 2006, vol. 1, pp. 5432-5435. https://doi.org/10.1109/IEMBS.2006.260613.

[23] E. Breda, M.K. Cavaghan, G. Toffolo, K.S. Polonsky and C. Cobelli, Oral glucose tolerance test minimal model indexes of beta cell function and insulin sensitivity, Diabetes, 2001, vol. 50, no. 1, pp. 150-158. https://doi.org/10.2337/diabetes.50.1.150.

[24] S.M. Lynch, B.W. Bequette, Model predictive control of blood glucose in type I diabetics using subcutaneous glucose measurements, Proceed. Am. Control Conf., 2002, vol. 5, pp. 4039-4043. https://doi.org/10.1109/ACC.2002.1024561.

[25] C. Dalla Man and R.A. Rizza, C. Cobelli, Meal simulation model of the glucose insulin system, IEEE Transactions on Biomed. Engineer, 2007, vol. 54, no. 10, pp. 1740-1749. https://doi.org/10.1109/TBME.2007.893506.

[26] A. Roy and R.S. Parker, Dynamic modeling of exercise effects on plasma glucose and insulin levels, J. Diabet. Sci Technol., 2007, vol. $1, \quad$ no. $3, \quad$ pp. 338-347. https://doi.org/10.1177/193229680700100305.

[27] J.T. Sorensen, A physiologic model of glucose metabolism in man and its use to design and assess improved insulin therapies for diabetes, Submitted to the Department of Chemical Engineering in partial fulfillment of the requirements for the Degree of Doctor of Science. Massachusetts Institute of Technology. Massachusetts, 1985, $556 \mathrm{p}$.

[28] R.S. Parker, F.J. Doyle and N.A. Peppas, A model based algorithm for blood glucose control in type I diabetic patients, IEEE Transact. Biomed. Engineer,. 1999, vol. 46, no. 2, pp. 148157. https://doi.org/10.1109/10.740877.

[29] R.S. Parker, F.J. Doyle, J.H. Ward and N.A. Peppas, Robust H [infinity] glucose control in diabetes using a physiological model, Am. Institute Chem. Engineers J., 2000, vol. 46, pp. 2537-2549. https://doi.org/10.1002/aic.690461220.

[30] C. Cobelli, G. Federspil, G. Pacini, A. Salvan and C. Scandellari, An integrated mathematical model of the dynamics of blood 
glucose and its hormonal control, Math. Biosci. 1982, vol. 58, pp. 27-60 https://doi.org/10.1016/0025-5564(82)90050-5.

[31] C. Cobelli and A. Mari, Validation of mathematical models of complex endocrine-metabolic systems. A case study on a model of glucose regulation. Med. Biol. Eng. Comput. 1983, vol. 21, no. 4, pp. 390-399. https://doi.org/10.1007/BF02442625.

[32] D.M. Eddy and L. Schlessinger, Archimedes: a trial validated model of diabetes, Diabetes Care, 2003, vol. 26, no. 11, pp. 30933101. https://doi.org/10.2337/diacare.26.11.3093.

[33] D.M. Eddy and L. Schlessinger, Validation of the archimedes diabetes model. Diabetes Care. 2003, vol. 26, no. 11, pp. 31023110. https://doi.org/10.2337/diacare.26.11.3102.

[34] S.G. Mougiakakou, K. Prountzou and K.S. Nikita, A Real Time Simulation Model of Glucose-Insulin Metabolism for Type I Diabetes Patients, 27th Annual International Conference of the Engineering in Medicine and Biology Society, 2005, pp. 298-301. https://doi.org/10.1109/IEMBS.2005.1616403.

[35] Yu. Lazarev, Modeling of processes and systems in MatLab, SPb.: Peter; Kiev: Publishing house group BHV, 2005, 512 p. (in Russian)

[36] Diabetes mellitus: diagnosis, treatment, prevention. Edited by I.I. Dedov and M.V. Shestakova, Moskow: LLC "Publishing house "Medical Information Agency", 2011, 808 p. (in Russian)
[37] N.A. Shirokova, Mathematical modeling of the insulin-glucose balance in the blood. Mathematical structures and modeling, 2002, vol. 10, pp. 106-115. (in Russian)

[38] V.V. Smirnov, Glucose monitoring system and insulin pumps. Therapist. 2009, vol. 3, pp. 31-35. (in Russian)

[39] Pathophysiology. Edited by N.N. Zaiko, Yu.V. Bytsya and N.V. Kryshtal, Kiev: VSI "Medicine", 2015, 744 p. (in Russian)

[40] I.P. Bolodurina, Yu.P. Ivanova (Lugovskova) and L.M. Antsiferova, Optimal Control of Glycemia Regulation Dynamics in Patients with Type I Diabetes Mellitus. Bulletin of the South Ural State University. Ser. Computer Technologies, Automatic Control, Radio Electronics, 2020, vol. 20, no. 4, pp. 144-154. https://doi.org/10.14529/ctcr200415. (in Russian)

[41] A.S. Ametov and L.L. Kamykina, Glycemic variability is the key to successfully managing type 2 diabetes in obesity. Russian medical journal. 2011, vol. 27, p. 1672. [Online]: Available:https://www.rmj.ru/articles/endokrinologiya/Variabelyn osty glikemii klyuch k uspeshnomu upravleniyu saharnym di abetom 2 tipa na fone_oghireniya/. [Accessed: Mart, 15, 2021]. (in Russian)

[42] D.N. Laptev, Insulin pump therapy with automatic shutdown of insulin delivery in response to hypoglycemia. Endocrinology problems, 2012, vo, 58, no. 3, pp. 70-74. (in Russian) 